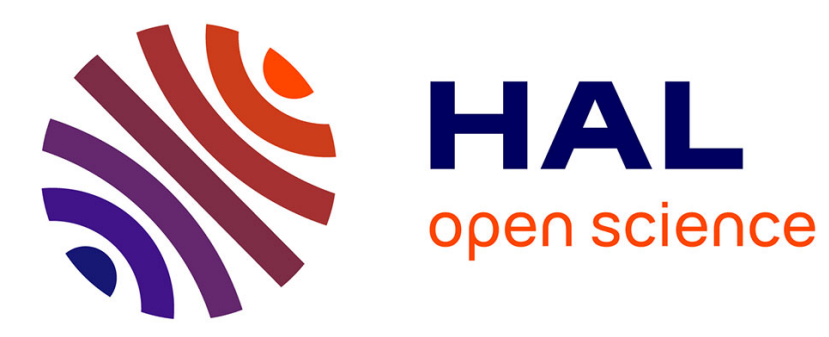

\title{
Scale filtered Euclidean medial axis and its hierarchy
}

Michal Postolski, Michel Couprie, Marcin Janaszewski

\section{To cite this version:}

Michal Postolski, Michel Couprie, Marcin Janaszewski. Scale filtered Euclidean medial axis and its hierarchy. Computer Vision and Image Understanding, 2014, 129, pp.89 - 102. 10.1016/j.cviu.2014.07.003 . hal-01228761

\section{HAL Id: hal-01228761 \\ https://hal.science/hal-01228761}

Submitted on 25 Oct 2016

HAL is a multi-disciplinary open access archive for the deposit and dissemination of scientific research documents, whether they are published or not. The documents may come from teaching and research institutions in France or abroad, or from public or private research centers.
L'archive ouverte pluridisciplinaire HAL, est destinée au dépôt et à la diffusion de documents scientifiques de niveau recherche, publiés ou non, émanant des établissements d'enseignement et de recherche français ou étrangers, des laboratoires publics ou privés. 


\title{
Scale Filtered Euclidean Medial Axis and its Hierarchy
}

\author{
Michał Postolski ${ }^{\mathrm{a}, \mathrm{b}}$, Michel Couprie ${ }^{\mathrm{a}}$, Marcin Janaszewski ${ }^{\mathrm{b}, *}$ \\ ${ }^{a}$ Université Paris-Est, LIGM, Equipe A3SI, ESIEE, \\ Cité DESCARTES BP 9993162 Noisy le Grand CEDEX, France \\ ${ }^{b}$ Eódź University of Technology, Institute of Applied Computer Science
}

\begin{abstract}
We propose an Euclidean medial axis filtering method which generates subsets of the Euclidean medial axis, where filtering rate is controlled by one parameter. The method is inspired by Miklos', Giesen's and Pauly's scale axis method which preserves important features of an input object from shape understanding point of view even if they are at different scales. Our method overcomes the most important drawback of the scale axis: the scale axis is not, in general, a subset of the Euclidean medial axis. It is even not necessarily a subset of the original shape. Moreover we propose a new method for the generation of a hierarchy of scale filtered Euclidean medial axes. We prove the correctness of the method. The methods and their properties are presented in 2D space but they can be easily extended to any dimension. Moreover, we propose a new methodology for the experimental comparison of medial axis filtering algorithms, based on five different quality criteria. This methodology allows one to compare algorithms independently on the meaning of their filtering parameter, which ensures a fair confrontation. The results of this confrontation with related previously introduced methods are included and discussed.
\end{abstract}

Keywords: Filtered medial axis, discrete scale axis, shape representation, image analysis, stability, hierarchy

\footnotetext{
${ }^{*}$ Corresponding author

Email address: janasz@kis.p.lodz.pl (Marcin Janaszewski)
} 


\section{Introduction}

The notion of medial axis has been introduced by Blum in the 60s [1]. The medial axis of an object $X$ is composed by the centres of the balls which are included in $X$ but which are not fully included in any other ball included in $X$. This set of points is, by nature, centred in the object with respect to the distance which is used to define the notion of ball.

In the literature, different methods have been proposed to compute the medial axis approximately or exactly, for instance methods relying on discrete geometry $[2,3,4,5]$, digital topology [6, 7], mathematical morphology [8], computational geometry [9, 10], partial differential equations [11], or levelsets [12]. In this work we focus on the discrete medial axis in $\mathbb{Z}^{n}$ based on the Euclidean metric.

The medial axis is a very useful representation of the object and plays a major role in shape analysis in numerous applications, for example object recognition, registration or compression. From the medial axis points and associated ball radii, one can exactly reconstruct the original shape. However it can be hard or even impossible to use this tool effectively without first dealing with some problems, especially in discrete spaces and with noisy objects.

Firstly, the medial axis in discrete spaces has not, in general, the same topology as the original object. Solutions to this problem have been proposed by several authors, for instance $[6,7,13]$. They use discrete homotopic transformations guided and constrained by the medial axis, to obtain an homotopic skeleton which contains the medial axis (see, Fig. 1). We do not consider these topological problems in the rest of the paper, and rely on this solution.

The second problem is the sensitivity of the Euclidean medial axis to small contour perturbations (see, for example, Fig. 1). In other words, the medial axis is not stable under small perturbations of a shape: modifying a shape slightly (for example in terms of Hausdorff distance) can result in substantially different medial axes. This is a major difficulty when the medial axis is used in practical applications (e.g. shape recognition). A recent survey which summarises selected relevant studies dealing with this topic is presented in [14]. This fact, among others, explains why it is usually necessary to add a filtering step (or pruning step) to any method that aims at computing the medial axis and when a nonreversible but simplified description of binary objects is of interest. 
(a)

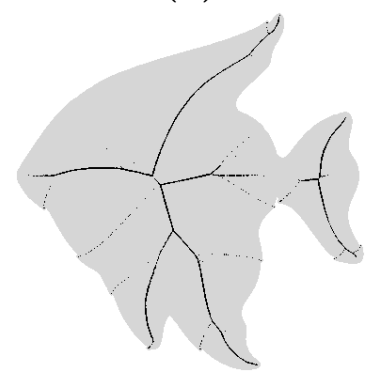

(b)

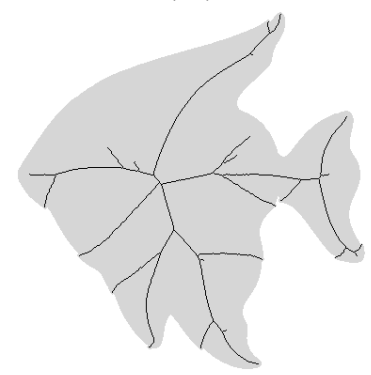

(c)

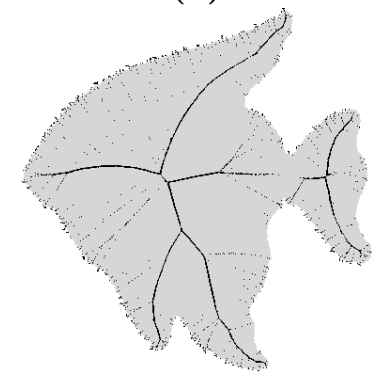

Figure 1: (a): a shape (in grey) and its Euclidean medial axis (in black); (b) the homotopic skeleton of the shape constrained by its Euclidean medial axis; (c) the same shape, but with small amount of noise added on the contour. The medial axis of the shape (c) is much more complicated than the medial axis of the shape (a).

The simplest strategy to filter the medial axis is to keep only points which are centres of maximal balls of at least a given diameter. Different criteria can be used to locally threshold and discard spurious medial axis points or branches: see $[15,16]$, for methods based on the angle formed by the vectors to the closest points on the shape boundary, or the circumradius of these closest points [17, 3].

In these methods, a local information (that is, geometric information extracted from a single medial ball) is compared to a global parameter value to determine the importance of the corresponding medial axis point. However, it is well known that this local filtering can lead to remove small branches which might be important for the shape understanding (see Fig. 2) especially for shapes with features at different scales [14].

A more complex criterion was proposed by [18]: the authors utilize information about ball importance in the shape with respect to all other balls by counting the number of object points inside a ball which are not covered by other balls. The medial axis point will be removed if the uncovered area of the corresponding ball is too small.

All these methods are based on a single parameter, furthermore they have the property that any filtered medial axis of a shape $X$ for a parameter value $\lambda$ is a subset of any filtered medial axis of $X$ for parameter value $\mu$, whenever $\lambda \geqslant \mu$. In other words, the set of all filtered medial axes of a shape forms a hierarchy.

In [19], the authors address the scale dependency issue and propose an 
(a)

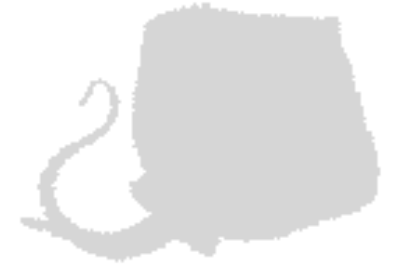

(b)

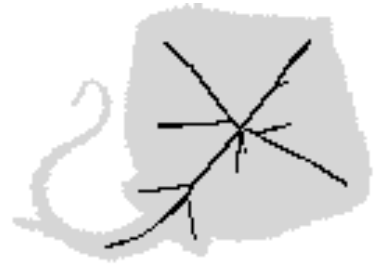

(c)

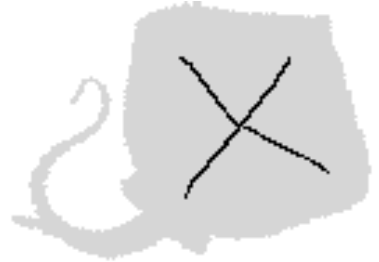

Figure 2: (a): a shape $X$ (in grey); (b): The filtered medial axis of $X$ (in black) calculated by using algorithm [5]. The medial axis is not sufficiently filtered in the middle of the shape. However, we already start to lose the tail; (c) A more filtered medial axis of $X$. Now, the middle of the shape is well filtered. However, we lost all information about the tail.

approach that puts in relation local information and regional information, that is, the status of a ball is only influenced by the one of neighbouring balls. Their method is based on the theory of the scale axis transform [20], which defines a whole family of medial representations at different levels of abstraction, called scale axis representations (see Fig. 3). For objects or scenes that include parts showing different scales, this method gives good results in many cases.
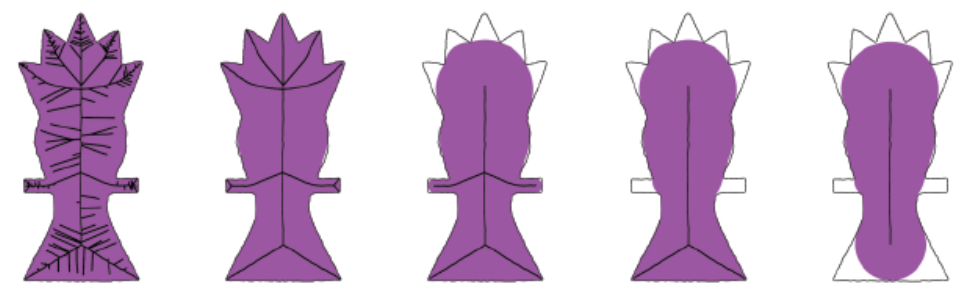

Figure 3: Different scale axes of the same object (contoured), using different values of the scale parameter. In pink, the part of the object reconstructed from the filtered axis.

However, the scale axis representation is not free of drawbacks. The most important one is that the scale axis is not necessarily a subset of the Euclidean medial axis (see Fig. 4), it is even not necessarily a subset of the original shape.

In this paper we propose a new method for the Euclidean medial axis filtering (see section 4). Our proposition is inspired by the scale axis method 
(see section 3). However, as result we obtain a filtered Euclidean medial axis, included in the exact one, instead of a set of points that is not necessarily a subset of the latter. Furthermore, our method produces axes that preserve important features for shape understanding, even if they are at different scales. Therefore, our algorithm overcomes the most important drawbacks noticed in previously presented methods following a similar approach. Moreover we propose a new, algorithm for the generation of a full hierarchy of medial axes with a proof of its correctness and an analysis of its properties (see section 5). The new algorithms work in arbitrary dimension. We evaluate experimentally their properties, and compare them with the previously introduced methods $[18,16]$. In addition, we propose a new methodology for comparison of medial axis filtering algorithms, which solves the problem of different interpretation of the algorithm parameters, and takes into account five different quality criteria (see section 6).

This article extends the paper [21], which was limited to the algorithm that computes a single filtered medial axis. Additionally, we introduce here a method for the generation of full medial axes hierarchies, we prove its correctness, we propose a new methodology for comparison of medial axis filtering algorithms, we report more experiments and discuss some properties of our new methods.

\section{Basic notions}

In this section, we recall some basic geometrical and topological notions for binary images [22, 23].

We denote by $\mathbb{Z}$ the set of integers, by $\mathbb{N}$ the set of nonnegative integers, and by $\mathbb{N}_{+}$the set of strictly positive integers. We denote by $E$ the discrete space $\mathbb{Z}^{d}$. A point $x$ in $E$ is defined by $\left(x_{1}, \ldots, x_{d}\right)$ with $x_{i}$ in $\mathbb{Z}$. Let $x, y \in E$, we denote by $d(x, y)$ the Euclidean distance between $x$ and $y$, that is, $d(x, y)=\left(\left(x_{1}-y_{1}\right)^{2}+\ldots+\left(x_{d}-y_{d}\right)^{2}\right)^{1 / 2}$. In practice, the squared Euclidean distance is used in order to avoid floating numbers. Let $Y \subset E$, we denote by $d(x, Y)$ the Euclidean distance between $x$ and the set $Y$, that is, $d(x, Y)=\min _{y \in Y}\{d(x, y)\}$. Let $X \subset E$ (the "object"), we denote by $D_{X}$ the map from $E$ to $\mathbb{R}_{+} \cup\{0\}$ which associates, to each point $x$ of $E$, the value $D_{X}(x)=d(x, \bar{X})$, where $\bar{X}$ denotes the complementary of $X$ (the "background"). The map $D_{X}$ is called the (Euclidean) distance map of $X$. Let $x \in E, r \in \mathbb{R}_{+}$, we denote by $B_{r}(x)$ the ball of radius $r$ centred on $x$, defined by $B_{r}(x)=\{y \in E, d(x, y)<r\}$. Notice that, for any point $x$ in $X$, 
the value $D_{X}(x)$ is precisely the radius of a ball centred on $x$ and included in $X$, which is not included in any other ball centred on $x$ and included in $X$.

Now, let us recall the notion of medial axis (see also $[24,7]$ ). Let $X \subseteq E$. A ball $B_{r}(x) \subseteq X$, with $x \in X$ and $r \in \mathbb{N}_{+}$, is maximal for $X$ if it is not strictly included in any other ball included in $X$. The medial axis of $X$, denoted by $\operatorname{MA}(X)$, is the set of the all couples $(x, r)$ such that $B_{r}(x)$ is a maximal ball for $X$.

Let $X \subset E, Y \subset X$, we denote by $R E D T_{X}(Y)$ the reverse Euclidean distance transform [18], defined by

$$
\operatorname{REDT}_{X}(Y)=\bigcup_{y \in Y} B_{D_{X}(y)}(y)
$$

The exact and unfiltered medial axis permits the exact reconstruction of the original object thanks to $R E D T$, more precisely we have $X=R E D T_{X}(M A(X))$.

\section{Discrete scale axis}

In this section, we adapt the notion of scale axis (see [19, 20]), originally introduced in the continuous space and implemented in a framework of unions of balls, to the case of discrete grids. We denote by $\mathbb{R}_{+}$the set of strictly positive real numbers. Let $X \subseteq E, x \in X, r \in \mathbb{N}_{+}$and $s \in \mathbb{R}_{+}$. The parameter $s$ is called the scale factor. We denote by $X_{s}$ the multiplicatively $s$-scaled shape, defined by $X_{s}=\bigcup_{(x, r) \in M A(X)} B_{r s}(x)$. For $s \geqslant 1$, we denote by $\operatorname{SAT}_{s}(X)$ the $s$-scale axis transform of $X$, defined by

$$
\operatorname{SAT}_{s}(X)=\left\{(x, r / s) \mid(x, r) \in M A\left(X_{s}\right)\right\} .
$$

The original algorithm to compute discrete scale axis, given by [19] in the framework of union of balls (UoBs), can be straightforwardly adapted to the case of $\mathbb{Z}^{d}$ as follows. First, calculate the Euclidean medial axis of $X$. To do so, we use an efficient algorithm presented in [18]. Then multiply the radius of each medial ball by the chosen scaling factor $s$.

In consequence, some small medial balls are covered completely by larger nearby balls since they are not important. On the other hand, small balls without larger balls in their neighbourhood are not covered and will be preserved.

The next step is to reconstruct the object $X_{s}$ based on scaled radius values. This reconstruction can be made efficiently by using the reverse 
Euclidean distance transform (see section 2). Computing the medial axis of $X_{s}$ achieves the simplification and $M A\left(X_{s}\right)$ will be free of all covered balls, since these do not touch the boundary any more and are thus no longer maximal. For $s=1$, the scale axis is identical to the unfiltered Euclidean medial axis. With increasing $s$, the scale axis gradually ignores less important features of $X$, leading to successive simplifications of $X_{s}$ and the scale axis structure.

The final step of the algorithm consists of rescaling the medial balls of $\operatorname{MA}\left(X_{s}\right)$ by a factor $1 / s$ to obtain the scale axis of $X$. Finally, a detailed discrete scale axis algorithm is provided in Algorithm 1.

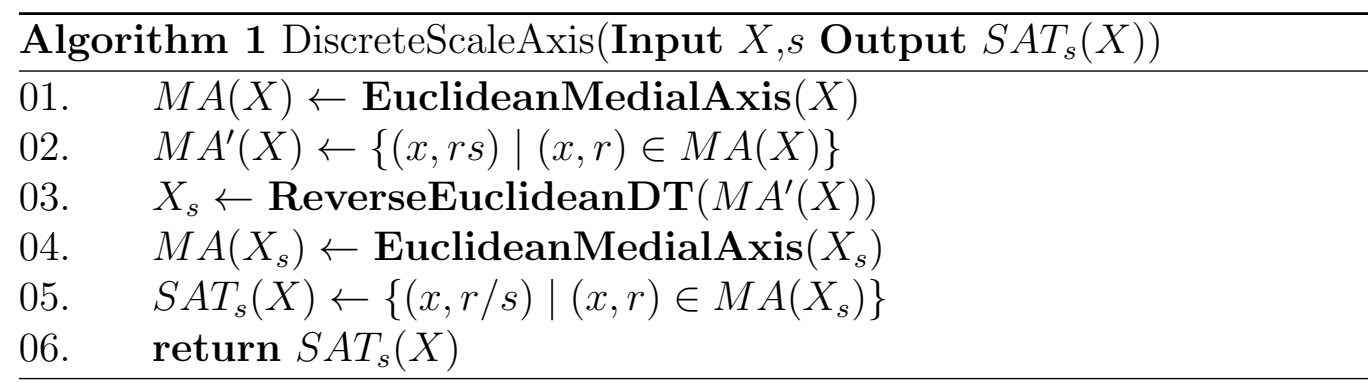

All four steps of DiscreteScaleAxis algorithm can be calculated in linear time in relation to $\# X$ (line 01), \#MA(X) (line 2), \# $X_{s}$ (lines 03-04) and $\# M A\left(X_{s}\right)$ (line 05) respectively, where $\# X$ stands for cardinality of $X$. Therefore, the computational complexity of the algorithm is $O\left(\# X_{s}\right)$.

\section{The scale filtered medial axis}

The crucial part of the method presented in the previous section, which is a source of problems $\left(\operatorname{SAT}_{s}(X) \nsubseteq \mathrm{MA}(X)\right)$, is the reconstruction part after medial balls scaling and the need for generating a new medial axis from the scaled object (see Fig. 4). On the other hand, at first sight, this is the most important part of the algorithm since the medial axis simplification occurs in this part.

To filter $\operatorname{MA}(X)$ by removing centres of unimportant medial balls one must avoid reconstruction and hold simplification property at the same time. To solve this problem, we remark that an efficient filtration may be obtained by only deciding which $\mathrm{MA}(X)$ points are not important and should be removed. Therefore, we do not have to generate a new object $X_{s}$ and its 
(a)

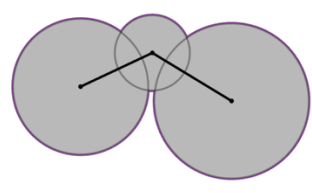

$s=1$ (b)

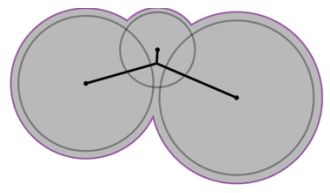

$s=1.1$ (c)

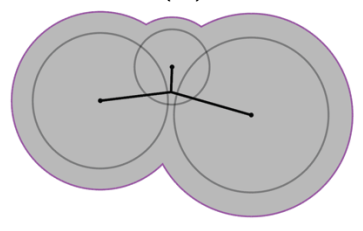

$s=1.3$

Figure 4: (a): a shape $X$ (in grey) and its Euclidean medial axis $M A(X)$ (in black); (b): the multiplicatively 1.1-scaled shape of $X$ and its 1.1-scaled axis; (c): the multiplicatively 1.3-scaled shape of $X$ and its 1.3 -scaled axis. In (b, c) we can see that scale axes are not subsets of $M A(X)$. In both cases, an additional branch even appears.

$\operatorname{MA}\left(X_{s}\right)$. In this way we can obtain a filtered Euclidean medial axis of $X$ which is a subset of $\operatorname{MA}(X)$.

This informal discussion motivates the following definition of the Scale Filtered Euclidean Medial Axis (SFEMA).

Let $x \in X, r \in \mathbb{N}_{+}$. We denote by $B_{r}^{X}(x)$ the intersection of $B_{r}(x)$ with $X$, that is, $B_{r}^{X}(x)=\{y \in X \mid d(x, y)<r\}$.

Definition 1. Let $X \subseteq E$, and $s \in \mathbb{R}, s \geqslant 1$. We denote by $S F E M A_{s}(X)$ the Scale Filtered Euclidean Medial Axis of $X$ defined by

$$
S F E M A_{s}(X)=\left\{(x, r) \in M A(X) \mid B_{r s}^{X}(x) \nsubseteq \bigcup_{(y, t) \in M A(X), t>r} B_{t s}^{X}(y)\right\} .
$$

Examples of $S F E M A_{s}(X)$ for different scale factors $s_{i}$, are shown in Fig. 5.

Below, we give an algorithm to compute $S F E M A_{s}(X)$ for a given object $X \subseteq E$.

Theorem 1. Algorithm SFEMA computes exactly the s-Scale Filtered Euclidean Medial Axis, as defined by Def. 1.

Proof. Consider any point $p$ in $X$ (after line 04). The assertion: "for all $k \in\{1, \ldots, i\}, d\left(x_{k}, p\right)>s r_{k}$ " holds true during the execution of the loop at line 06. After execution of this loop (line 07) we know that for all $k \in\{1, \ldots, i-1\}, d\left(x_{k}, p\right)>s r_{k}$, and $d\left(x_{i}, p\right) \leqslant s r_{i}$. Thus, at line 08 , we know that $p$ belongs to $B^{X}\left(x_{i}, r_{i} s\right)$ and that it does not belong to any 


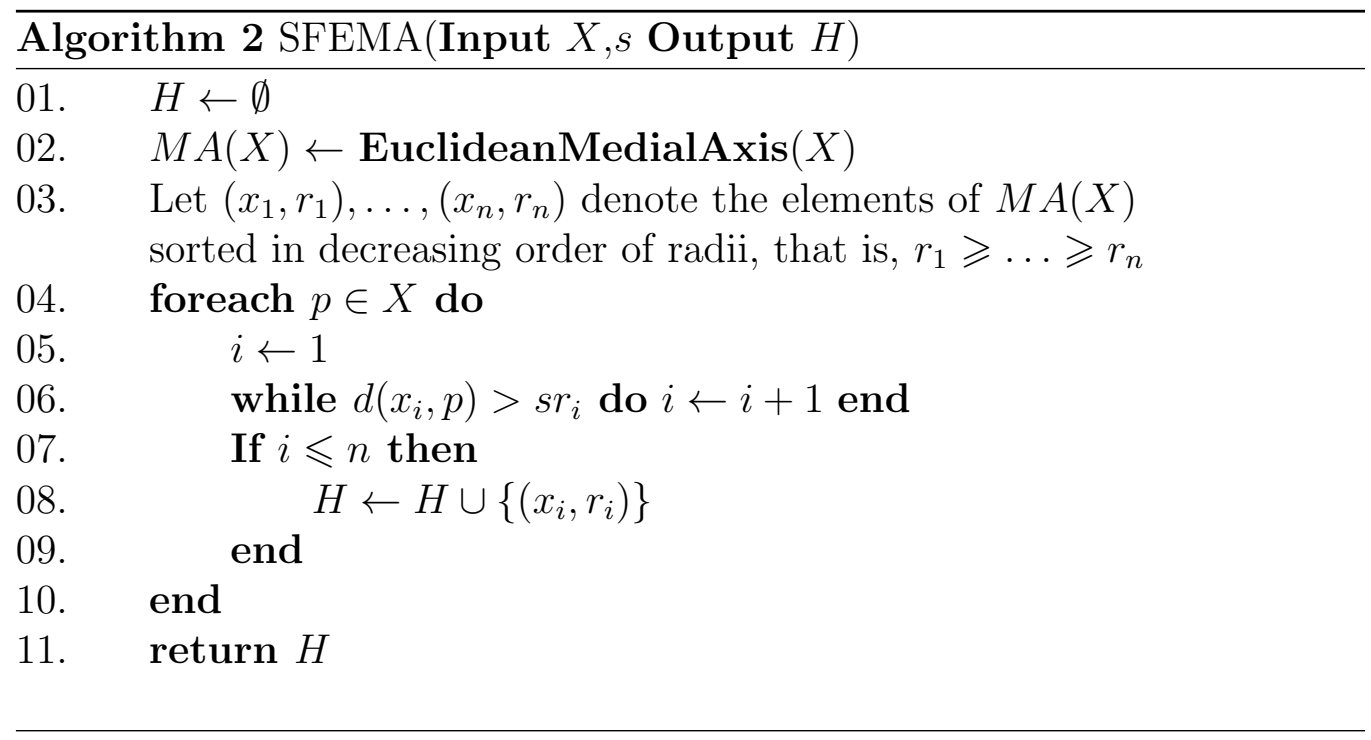

ball $B^{X}\left(x_{k}, r_{k} s\right)$, with $r_{k}>r_{i}$. From this we deduce that $\left(x_{i}, r_{i}\right)$ belongs to $S F E M A_{s}(X)$.

This proves that all detected pairs indeed belong to $S F E M A_{s}(X)$. Now consider any pair $(x, r)$ of $S F E M A_{s}(X)$, there exists at least one point $p$ in $B^{X}(x, r s)$ that is not included in $\left.\bigcup_{(y, t) \in M A(X), t>r} B^{X}(y, t s)\right\}$. As all points $p$ of $X$ are considered by the algorithm (line 04 ), the pair $(x, r)$ will be detected.

The algorithm in line 02 performs sorting of medial axis elements, linearly in time using a counting sort [25]. In the following lines the algorithm performs two loops. The first one starts in line 04 and does \# $X$ iterations. The next, nested loop, starts in line 06 and in worst the case performs $\# M A(X)$ iterations. Summarizing, computational complexity of SFEMA is $O(\# X \# M A(X))$.

Let us analyse the properties and the major differences between the Miklos's [19] $s$-scale axis and our $s$-scale filtered Euclidean medial axis. The most important property is that $S F E M A_{s}(X)$ consists of $M A(X)$ points only, that is, for all $s \geqslant 1: \operatorname{SFEMA}(X) \subseteq M A(X)$. This property (inclusion property, for short) is essential in many applications of the medial axis. In Fig. 4 we have shown an example of the Miklos's scale axis where an additional branch even appears after filtering. Fig.6 shows another problem. The scale axis is too much simplified, loses important features of the object and is not included in the object. However, $s$-scale filtered medial axis holds 
(a)

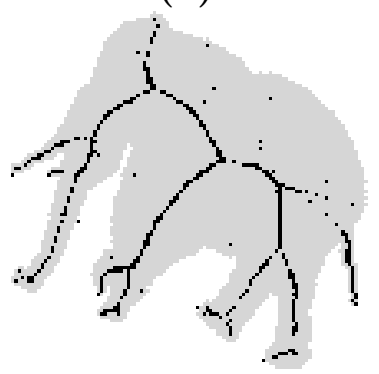

(c)

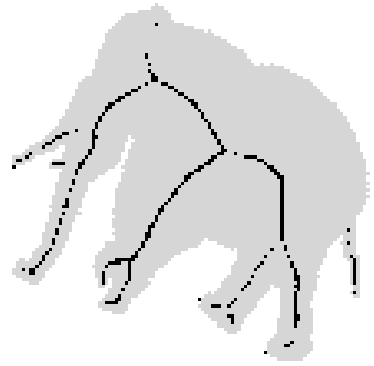

(b)

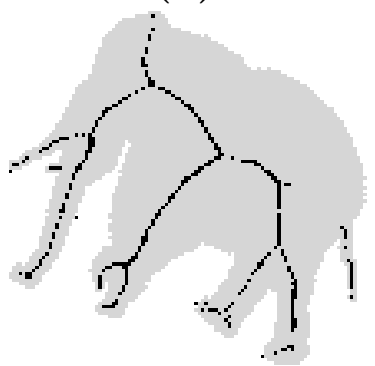

(d)

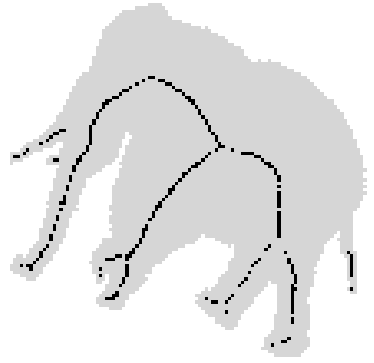

Figure 5: (a): a shape $X$ (in grey) and its Euclidean medial axis (in black); (b, c, d): the same shape and its $S F E M A_{1.1}(X), S F E M A_{1.4}(X), S F E M A_{1.6}(X)$, respectively. In all cases the elephant's tail, trunk, tusks and legs were considered as important and were not removed.

inclusion property and permits to reconstruct most of the original object.

The second interesting property relies on the notion of s-scaled ball. If we want to simplify the object, using Miklos's scale axis, for example by removing a medial ball $B_{r}(x), x \in X$, the scale factor should be big enough in order that ball $B_{r s}(x)$ is included in one of other medial balls, that is, $B_{r s}(x) \subset B_{r s}(y), y \in X$ (see Fig.7c), or in a union of such balls. In our algorithm, since we use notion of s-scaled ball, we only test inclusion inside $X$ (see Fig.7b). This allows us to use a smaller scale factor. Therefore, we have a better ability to control the resulting $s$-scale filtered Euclidean medial axis.

\section{Algorithm for computing the hierarchical scale medial axis}

Intuitively, the greater the scale parameter $s$ used in $S F E M A$, the smaller the filtered axis. Moreover for each object $X, S F E M A$ produces a family of 
(a)

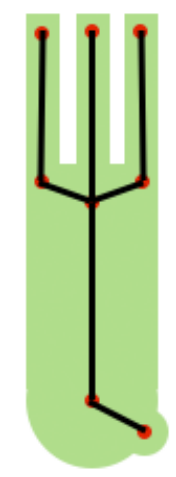

original shape (b)

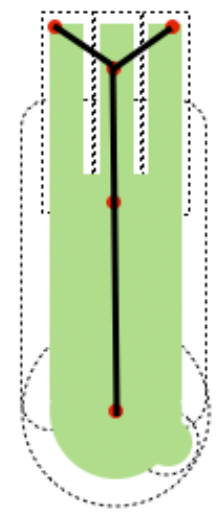

$s=1.3$ (c)

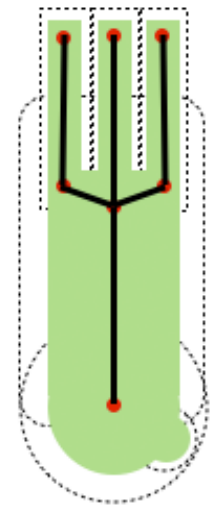

$s=1.3$

Figure 6: (a): a set $X$ (in green) and its $M A(X)$ (in black); (b): the 1.3-scale axis of $X$; (c): the 1.3-scale filtered medial axis of $X$.

(a)

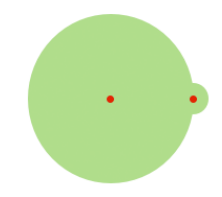

original object (b)

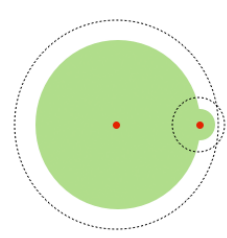

$s=1.1$ (c)

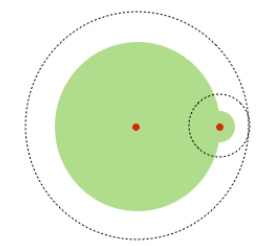

$s=1.3$

Figure 7: (a): a set $X$ (in green) and its $M A(X)$ (red dots); (b): multiplicatively scaled medial balls. The smaller ball is not fully covered by the bigger one after scaling. In scale axis representation both balls will be preserved. However, the bigger ball includes the smaller one inside set $X$. Therefore, the smaller ball will not exist in $\operatorname{SFEMA}_{s}(X)$; (c): multiplicatively scaled medial balls. The smaller ball is included in the bigger one.

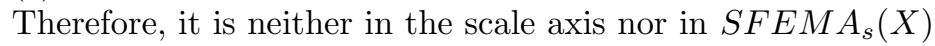

axes that are all subsets of $M A(X)$. This suggests that these axes could be nested into each other. In this section, we formalize this property, we show that it is not always true with the axes defined by $S F E M A$, and we propose a new algorithm to compute directly a family of nested filtered axes. 
Definition 2. We denote by $M A_{s}(X)$ the filtered medial axis of $X \subset \mathbb{Z}^{n}$ for $s \in S \subseteq \mathbb{R}_{+}$, where $S$ represents the set of all possible values of parameter $s$ of the considered filtering algorithm. The family $H=\left\{M A_{s}(X) \mid s \in S\right\}$ is a hierarchy if $\forall s_{1}, s_{2} \in S \mid s_{2}>s_{1} \Rightarrow M A_{s_{2}}(X) \subseteq M A_{s_{1}}(X) \subseteq M A(X)$.

Unfortunately, SFEMA does not hold hierarchy property, it means that there is $X \subset \mathbb{Z}^{n}$ such that $\left\{\operatorname{SFEMA} A_{s}(X) \mid s \geq 1\right\}$ is not a hierarchy. A counter-example to the hierarchy property is presented in the Fig. 8. This fact motivates the following definition:

Definition 3. Let $X \subset \mathbb{Z}^{n}$ and $(x, r) \in M A(X)$. We denote by $\Psi_{X}: M A(X) \rightarrow[1, \infty) \cup\{\infty\}$ the scale map of $X$ defined by

$$
\Psi_{X}(x, r)=\min \left\{t \geq 1 \mid \forall s \geq t,(x, r) \notin \operatorname{SFEMA} A_{s}(X)\right\}
$$

We see that thresholding the map $\Psi_{X}$ at a given level $s$ gives us a filtered axis. By construction, all these axes form a hierarchy.

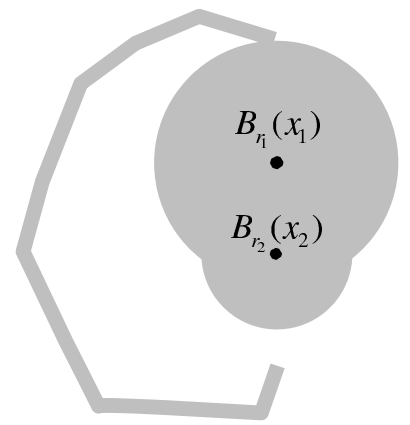

(a)

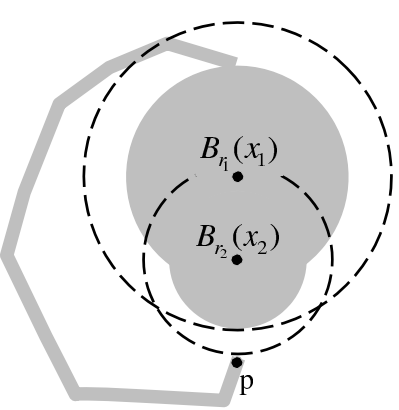

(b)

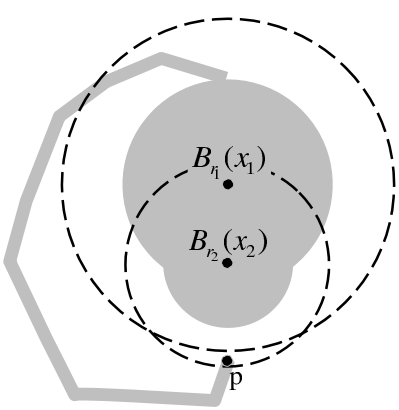

(c)

Figure 8: (a): a shape $X$ (in grey) and two maximal balls $B\left(x_{1}, r_{1}\right), B\left(x_{2}, r_{2}\right) \in X$; (b): dashed circles represent scaled balls by $s=1.4$. One can observe that $B^{X}\left(x_{2}, r_{2} 1.4\right) \subset$ $B^{X}\left(x_{1}, r_{1} 1.4\right)$, so $\left(x_{2}, r_{2}\right) \notin S F E M A_{1.4}(X) ;(c)$ : dashed circles represent scaled balls by $s=1.6$, now $p \in B^{X}\left(x_{2}, r_{2} 1.6\right)$ and $p \notin B^{X}\left(x_{1}, r_{1} 1.6\right)$, so $\left(x_{2}, r_{2}\right) \in S F E M A_{1.6}(X)$, which finally leads to the conclusion that $S F E M A_{s}(X)$ does not hold hierarchy property.

Now, let us introduce algorithm 3, which calculates the scale map. It is easier to understand and prove than our final one, but less efficient.

To facilitate the understanding of algorithm 3 we present its way of working based on fig. 5 .

Before proving the validity of the algorithm, let us first remark that, in the definition of $\Psi$, it is not necessary to consider all possible values of $t$ or 


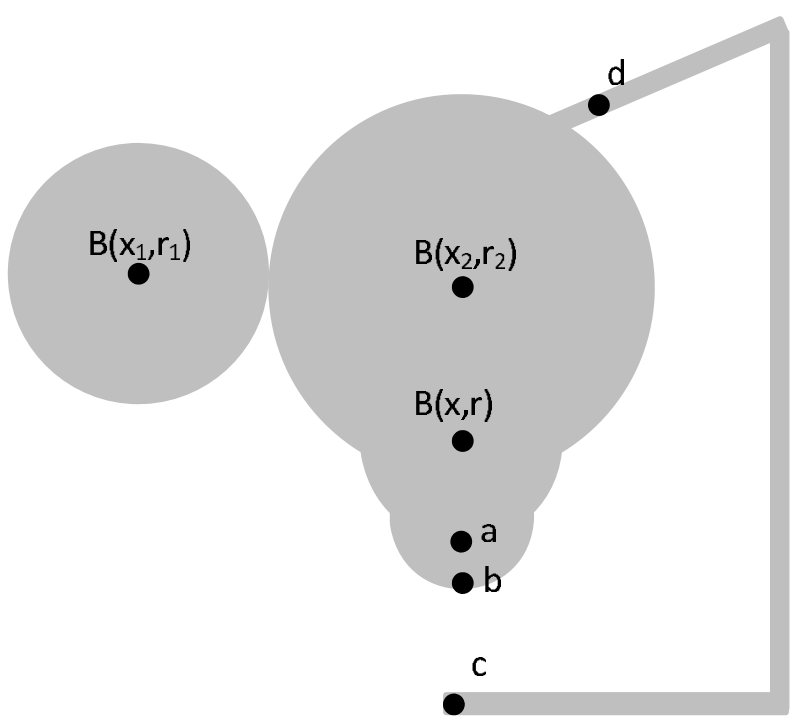

Figure 9: Presents the idea of the algorithm 3 way of working. Assume that the algorithm calculates $\Psi(x, r)$. For a point $a$ it calculates $t_{\min }$ as a minimum of $d\left(a, x_{i}\right) / r_{i}$ for all balls $B\left(x_{i}, r_{i}\right)$ (shortly $B_{i}$ ) greater than $B(x, r)$ (shortly $B$ ) in lines 05-07. In our example only two maximal balls are greater than $B: B_{1}$ and $B_{2}$. We have $d\left(a, x_{1}\right) / r_{1}=2.097$, $d\left(a, x_{2}\right) / r_{2}=1.34$. So $t_{\min }=1.34$. It means that during scaling the ball $B_{2}$ covers the point $a$ as the first of all maximal balls greater than $B$. Moreover the minimum cover scale $s$ such that $a \in s B_{2}$ is equal to 1.34 . Then the algorithm, in line 09, checks if ball $B$ reaches the point $a$ for smaller scaling factor than $B_{2}$ (smaller than $t_{\text {min }}$ ). In our case $d(x, a) / r=1.06$. So point $a$ belongs to a sum of balls greater than $B$ for minimum cover scale $s=1.34$ and it belongs to the ball $B(x, r * 1.34)$, thus if $\Psi(x, r)<1.34$ the algorithm updates $\Psi(x, r)$ in line 10. Similarly for the point $b$ we have $d(x, b) / r=$ $1.06, d\left(x_{1}, b\right) / r_{1}=2.39, d\left(x_{2}, b\right) / r_{2}=1.54$, so $t_{\min }=1.54$ and $\Psi(x, r)$ is set to 1.54 if less than 1.54. The situation is different for the point $c: d(x, c) / r=2.64, d\left(x_{1}, c\right) / r_{1}=3.15$, $d\left(x_{2}, c\right) / r_{2}=2.17$, it means that $t_{\min }=2.17<d(x, c) / r$ so when the union of scaled by 2.17 balls greater than $B$ reaches point $c$, it does not belong to $B(x, r * 2.17)$ so the algorithm does not update $\Psi(x, y)$. The same situation occurs for point $d$, when reached by scaled $B_{2}$ it does not belong to scaled $B$ so $\Psi(x, y)$ is not updated. In the example $\Psi(x, y)=2.17$ and the maximum $t_{m i n}$ over all points from $X$ is obtained for $b$. 


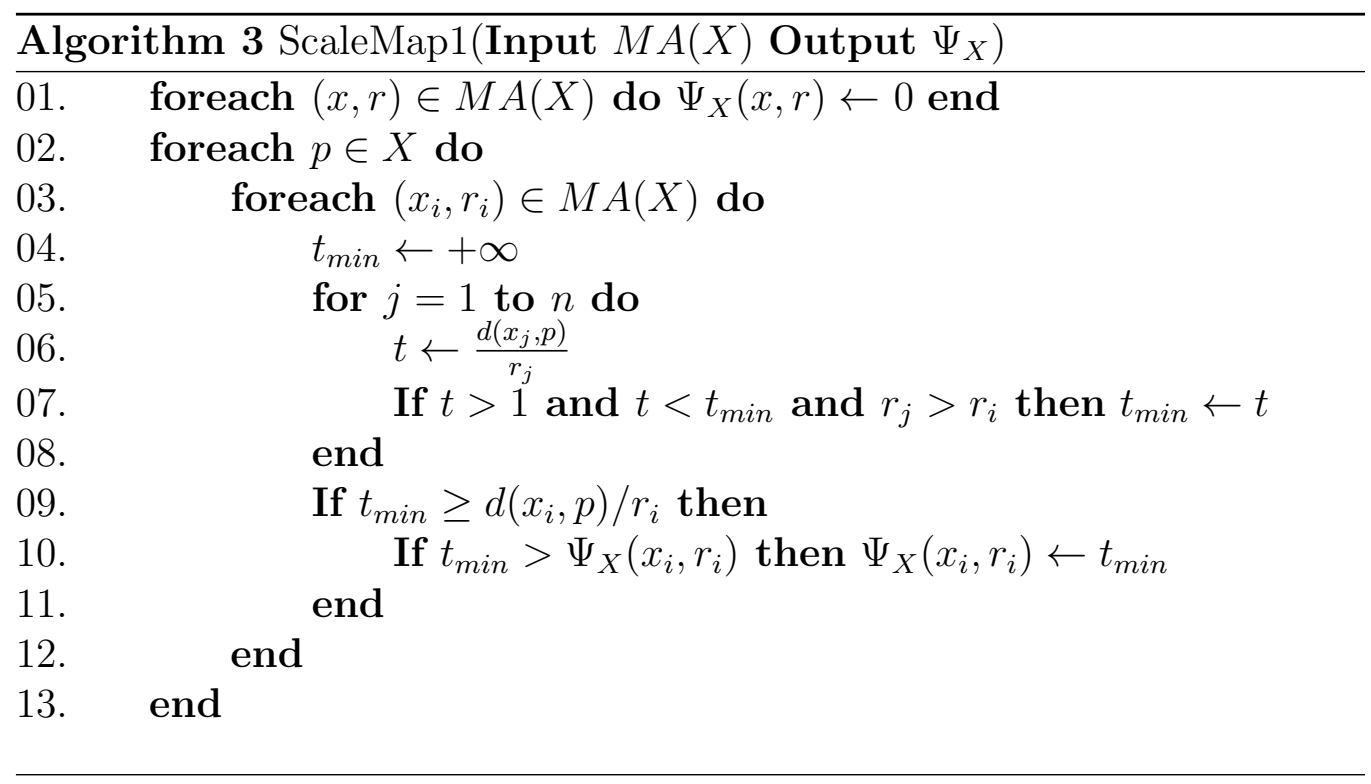

$s$, but only the ones of the form $\frac{d(x, p)}{r}$, with $p \in X$ and $(x, r) \in M A(X)$, and $+\infty$. Thus, we define:

$$
\begin{gathered}
S_{p}=\left\{s=\frac{d\left(x_{i}, p\right)}{r_{i}} ;\left(x_{i}, r_{i}\right) \in M A(X) \text { and } s>1\right\} \cup\{+\infty\} \\
S=\bigcup_{p \in X} S_{p}
\end{gathered}
$$

And we have:

$$
\Psi_{X}(x, r)=\min \left\{t \in S \mid \forall s \in S, s \geq t,(x, r) \notin S F E M A_{s}(X)\right\}
$$

Let $r_{1}$ be the radius of the biggest ball in $M A(X)$, that is, $r_{1}=\max \left\{r_{i} \mid\right.$ $\left.\left(x_{i}, r_{i}\right) \in M A(X)\right\}$. Now, for any $p$ in $X$ and any $(x, r) \in M A(X)$ let us define

$$
E(p, r)= \begin{cases}\min \left\{t \in S_{p} \mid \forall s \in S, s \geqslant t, p \in \bigcup_{r_{j}>r} B\left(x_{j}, s r_{j}\right)\right\} & \text { if } r<r_{1} \\ +\infty & \text { otherwise }\end{cases}
$$


For a point $p$ and a radius $r, E(p, r)$ is the minimum scale for which $p$ is covered by a scaled medial ball of radius greater than $r$ (minimum cover scale).

Moreover let us define:

$$
X(x, r)=\{p \in X: E(p, r) \geq d(p, x) / r\}
$$

For a medial ball $(x, r), X(x, r)$ represents the set of all points $p$ of $X$ such that the minimum cover scale $E(p, r)$, applied to the ball $(x, r)$, suffices to cover $p$.

From the last definition we have:

$$
X(x, r)=\left\{p \in X: p \in B^{X}(x, r E(p, r))\right\}
$$

\section{Proposition 1.}

For any $(x, r)$ in $M A(X)$, we have $\Psi_{X}(x, r)=\max _{p \in X(x, r)} E(p, r)$.

Proof.

Let $r=r_{1}=\max \left\{r_{i} \mid\left(x_{i}, r_{i}\right) \in M A(X)\right\}$. Then we have $X(x, r)=X$, $E(p, r)=+\infty$ for any $p \in X$ and $\Psi_{X}(x, r)=+\infty$. From now on, we suppose that $r<r_{1}$.

Let us set $\hat{p}=\arg \max _{p \in X(x, r)} E(p, r)$ and $\hat{t}=E(\hat{p}, r)$. Hence, we have $\hat{t}=\max _{p \in X(x, r)} E(p, r)$. So, directly from (2) we have that $\forall p \in X(x, r), p \in$ $\bigcup_{r_{j}>r} B\left(x_{j}, \hat{t} r_{j}\right)$, thus $\forall s \in S, s \geq \hat{t}, X(x, r) \subseteq \bigcup_{r_{j}>r} B^{X}\left(x_{j}, s r_{j}\right)$. Moreover, by the very definition of $\hat{t}, \forall s \in S, s<\hat{t}: \hat{p} \notin \bigcup_{r_{j}>r} B\left(x_{j}, s r_{j}\right)$. Thus, combining the last two expressions we obtain:

$$
\begin{gathered}
\max _{p \in X(x, r)} E(p, r)=\min \left\{t \in \bigcup_{p \in X(x, r)} S_{p} \mid \forall s \in S, s \geq t\right. \\
\left.X(x, r) \subseteq \bigcup_{r_{j}>r} B\left(x_{j}, s r_{j}\right)\right\}
\end{gathered}
$$

From (4) and the definition of $\hat{t}$, we have:

$$
\forall s \in S, s>\hat{t}, X(x, r) \subseteq B^{X}(x, s r)
$$

Now let us take any $p \in X \backslash X(x, r)$. From (3) we have $E(p, r)<d(p, x) / r$. So, by definition of $E(p, r)$, for any $s \in S$ such that $p \in B^{X}(x, r s)$, we have 
$p \in \bigcup_{r_{j}>r} B^{X}\left(x_{j}, s r_{j}\right)$. In other words:

$$
\forall s \in S, B^{X}(x, r s) \backslash X(x, r) \subseteq \bigcup_{r_{j}>r} B^{X}\left(x_{j}, s r_{j}\right)
$$

Finally from (5), (6) and (7) we have:

$$
\begin{aligned}
& \max _{p \in X(x, r)} E(p, r)= \min \{t \in S \mid \forall s \in S, s \geq t, \\
&\left.B(x, s r) \subseteq \bigcup_{r_{j}>r} B\left(x_{j}, s r_{j}\right)\right\}=\Psi(x, r)
\end{aligned}
$$

Theorem 2. Algorithm ScaleMap1 calculates exactly scale map, as defined in the Def. 3

Proof. Algorithm ScaleMap1, for a medial ball $B\left(x_{i}, r_{i}\right)$ and a point $p \in X$ in lines 05-07 calculates $t_{\text {min }}$ equal to $E\left(p, r_{i}\right)$ defined by $(2)$.

Then in line 09 it checks whether $p$ belongs to $X\left(x_{i}, r_{i}\right)$, see (3). Thus, we have that the algorithm (line 10) calculates $\max _{p \in X\left(x_{i}, r_{i}\right)} E\left(p, r_{i}\right)$.

By proposition 1, the algorithm calculates correctly $\Psi\left(x_{i}, r_{i}\right)$, for each $i$.

Now, let us present a variant of the previous algorithm, that computes the same result but with a lower time complexity.

Let us define a new medial axis filtering strategy, which holds hierarchy property, based on the scale map calculated by algorithm 4 .

Definition 4. Let $X \subset \mathbb{Z}^{n}, s>1$ and $\Psi_{X}$ is a scale map of $X$. We define by $H S M A_{s}(X)$ the hierarchical scaled medial axis of $X$ by the following formula:

$$
H S M A_{s}(X)=\left\{(x, y) \in M A(X) \mid \Psi_{X}(x, r)>s\right\}
$$

In conclusion, we have the following property, which is straightforwardly consequence of definitions 2 and 4 .

Theorem 3. Let $X \subset E$. The family $H=\left\{H S M A_{s}(X) \mid s>1\right\}$ is a hierarchy. 


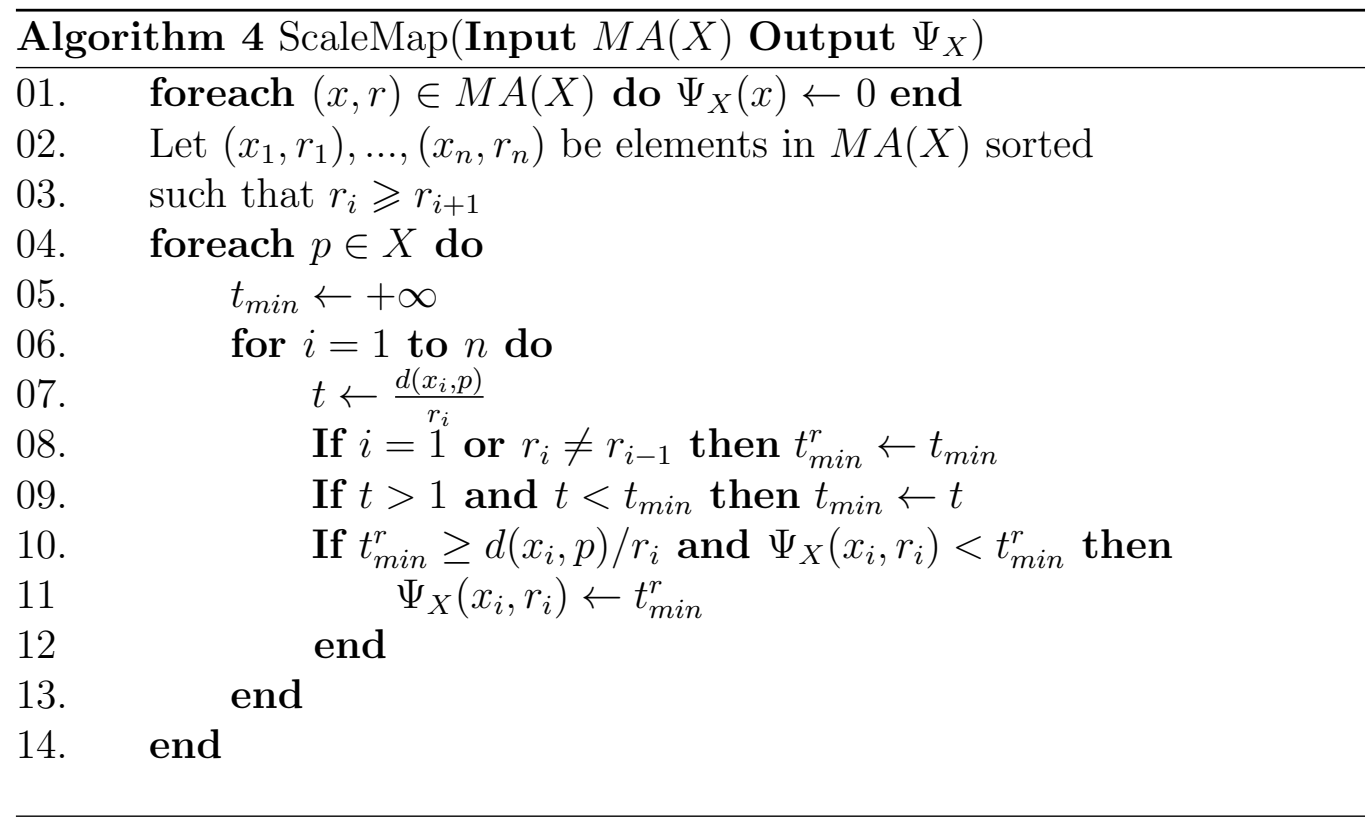

The computational complexity of algorithm 4 is the same as algorithm 2, that is, $O(|X||M A(X)|)$. The algorithm in line 02 performs sorting of medial axis elements. It is possible to implement sorting algorithm in linear time with the use of a counting sort. In the following lines the algorithm performs two loops, the second nested in the first. The first one starts in line 04 and does $|X|$ iterations. The next nested loop starts in line 06 and performs $|M A(X)|$ iterations, where $|M A(X)| \ll|X|$ (usually).

\section{Experiment methodology and results}

In this section, we compare qualitatively and quantitatively properties of five medial axis filtering algorithms. In the comparison we include algorithms that operate in the same framework as ours: discrete $\lambda$-medial axis (DLMA) [5], the Euclidean medial axis filtered with the use of bisector function (BFMA) [16], filtering based on medial ball radius (RFMA) [18], filtering based on ball covering (CFMA) [18] and a filtering method proposed in this work: the hierarchical scaled medial axis (HSMA).

In this study, we do not consider the topology preservation issue, as none of these methods provide a guarantee of preserving topology. A classical way to obtain topologically correct skeletons from a sparse medial axis, consists 
of performing an homotopic thinning of the shape with the constraint of preserving the medial axis points $[6,7,13]$.

In our experiments we use six selected shapes from the Kimia's database [26], that are representative of the whole set. Four of the selected objects: "Ray", "Elephant", "Camel" and "Fountain", have many important features at different scales, in contrast to the two other ones: "Classic" and "Misk" (see Fig. 10).

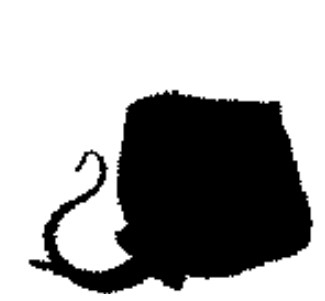

(a) Ray

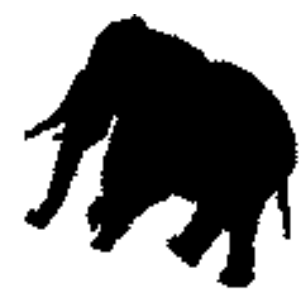

(b) Elephant

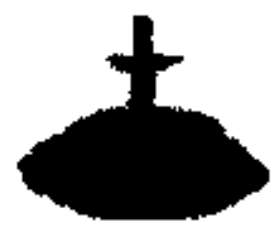

(c) Fountain

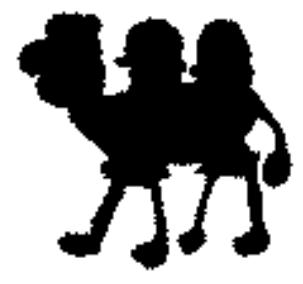

(d) Camel

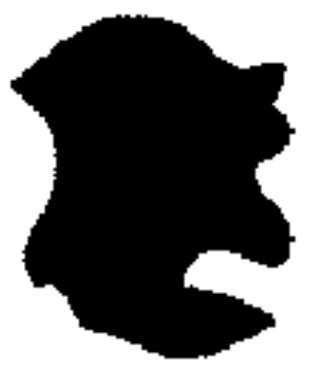

(e) Misk

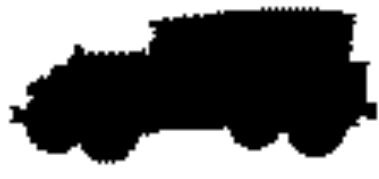

(f) Classic

Figure 10: The selected 6 representative shapes of Kimia's database used in our experiments.

It is difficult to compare methods, all based on a single filtering parameter, but with quite different meanings of this parameter. Due to this fact, comparing the results of two methods for the same parameter value does not make sense. Therefore, we have to find some strategies to ensure a fair comparison between methods, based on criteria that do not depend on their particular way of working. Furthermore, we want these criteria to be related with actual concerns met in real-world applications.

Several criteria have already been proposed in the literature. Chaussard [27] defined the so-called visual quality factor which is a combination of reconstruction error with information derived from the skeleton of the shape, namely, the number of skeleton points. Van Eede et. al [28] propose 
a solution based on weighted reconstruction error, combined with skeleton complexity defined as number of skeleton branches. However, the quality of the medial axis depends of the reconstruction weight which need to be set arbitrarily. Setting this parameter properly is not obvious.

In the following subsections, we propose a comparison methodology that is based on five different criteria. In each case, we give both the definition and the intuitive meaning of the criterion, and we report the results of our experiments.

\subsection{The reconstruction error}

Let $X \subset \mathbb{Z}^{n}, Y \subset X$, we denote by $R E D T_{X}(Y)$ the reverse Euclidean distance transform [18], defined by

$$
\operatorname{REDT}_{X}(Y)=\bigcup_{y \in Y} B(y, r)
$$

For the exact and unfiltered $\mathrm{MA}(X)$ we have $X=R E D T_{X}(M A(X))$. However, this property is no longer true if we consider filtered medial axes e.g. HSMA, DLMA, BFMA, RFMA or CFMA. Therefore, it is interesting to measure how much information about the original object is lost when we perform filtering. We define

$$
R_{X}(Y)=\frac{\left|\left(X \cup R E D T_{X}(Y)\right) \backslash\left(R E D T_{X}(Y) \cap X\right)\right|}{\left|X \cup R E D T_{X}(Y)\right|}
$$

We call $R_{X}(Y)$ the (normalised) residuals of $Y$. Residuals give us a numerical evaluation of reconstruction error. Now we can set $Y$ to different filtered medial axes, e.g. by using different methods or filtering parameter values, and then evaluate which one filtration is the best in respect of their ability to allow for a faithful reconstruction of the object. The result $R_{X}(Y)$ is a real value between 0 (perfect reconstruction) and 1 (bad reconstruction).

Figure 11 presents DLMA, BFMA, HSMA, RFMA and CFMA of a chosen shape, extracted for several values of normalised residuals. Note that we compare the results of methods for approximately equal values of their residuals, rather than for equal values of their parameters. One can observe, in figure 11, that DLMA loses representation of tail even for low values of residuals. On the other hand, BFMA preserves representation of tail but it also preserves unimportant balls on the border of the object. Such balls may represent noise. HSMA preserves representation of tail even for high value of 
residuals. Moreover it successfully removes unimportant balls even for low values of residuals. RFMA loses tail even for low value of residuals and it preserves many unimportant balls. CFMA successfully removes unimportant balls but loses some representation of tail for high residuals.

For the sake of paper size we have not included results for other objects from figure 10. Interested reader can find the results in [29] and draw similar conclusions as from Figure 11.
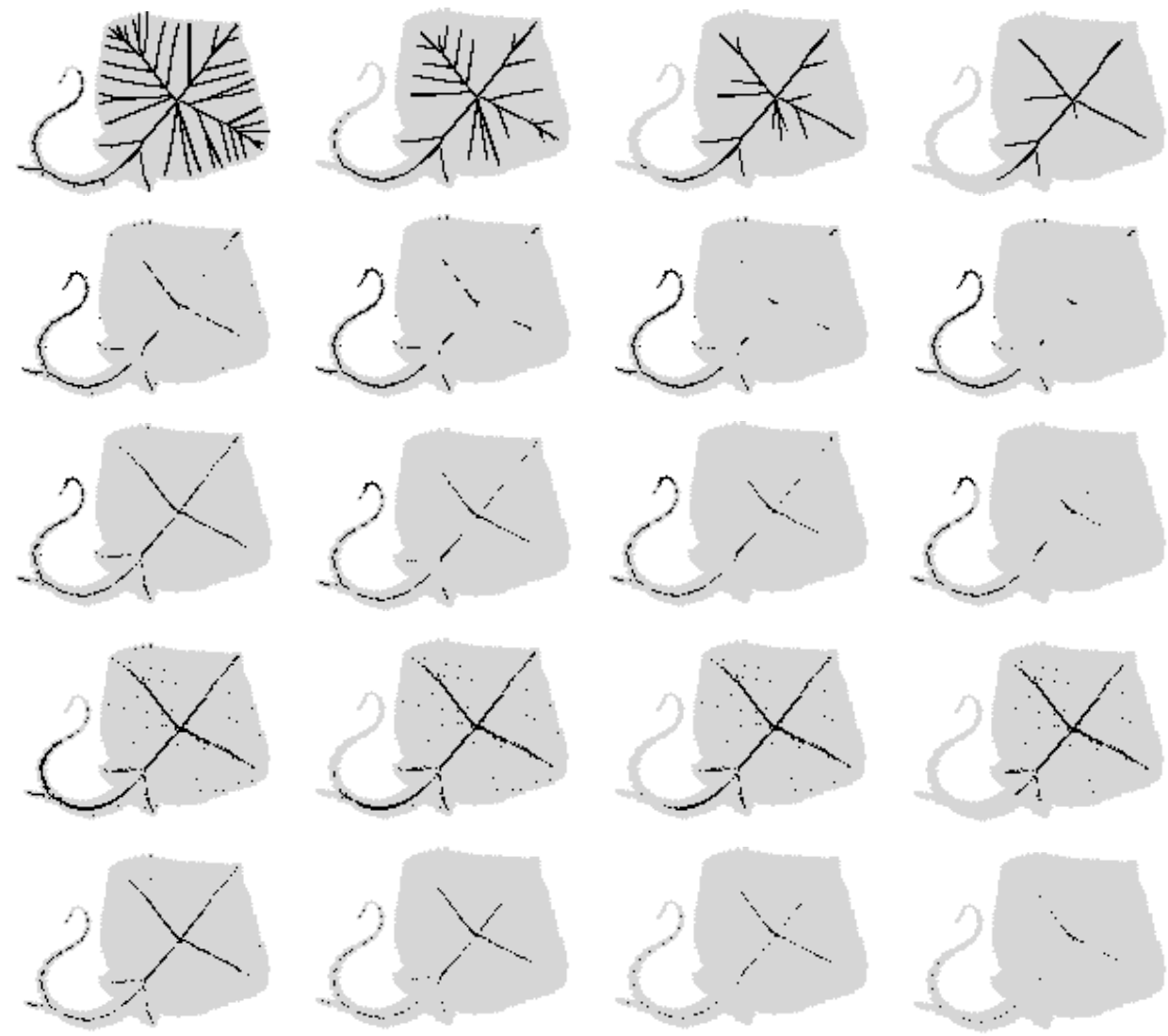

Figure 11: Medial axes (in black) superimpose to input object (in grey). Consecutive rows (from up to the bottom) contain results for DLMA, BFMA, HSMA, RFMA and CFMA respectively. Columns contain results for different values of normalised residuals: 0.01 , $0.03,0.05,0.1$ respectively. 


\subsection{The normalised medial axis size}

We call $N S_{X}(Y)$ the normalised medial axis size, defined as the ratio of the number of medial axis points to the number of object points:

$$
N S_{X}(Y)=|Y| /|X|
$$

Now we can compare the normalised residuals obtained for the same $N S$, using different methods. Intuitively, this allows us to judge of the importance (on average), with regard to reconstruction, of the medial axis points that are kept by the filtering method.

In Fig. 12 we show the normalised residuals $R$ as a function of normalised medial axis size $N S$ for different shapes and different filtering methods. The results shows that the CFMA obtains the smallest residual values for the very small normalised sizes of filtered medial axis. When the size of the medial axis is large enough the CFMA behaves similarly to BFMA and HSMA. It means that CFMA, BFMA and HSMA, at the first place remove points which not affect the reconstruction error. In contrast, with RFMA and DLMA, some important (for reconstruction) points are removed even for very large size of medial axes.

\subsection{The border dissimilarity}

From the shape understanding point of view, preserving visually important segments and removing unimportant ones, at the same time, is even more important than achieving very good reconstruction property. Based on the two previous criteria only, is hard to take into account the negative impact of the removal of a visually important feature that has a relatively small size. Therefore, in this section, we introduce another comparison strategy which allows us to observe how different filtering strategies preserve visually important features.

Globally, the filtering algorithms will be tested by measuring the "difference" between the border of an object $X$, and the border of the reconstructed shape after filtering. The "difference" will be measured using the dissimilarity measure commonly used in replacement of the Hausdorff distance, because of its lower sensitivity to outliers, and defined as follows:

Let $X, Y$ be two subsets of $\mathbb{R}^{n}$. We set

$$
D(X \mid Y)=\frac{1}{|X|} \sum_{x \in X} \min _{y \in Y}\{d(x, y)\}
$$




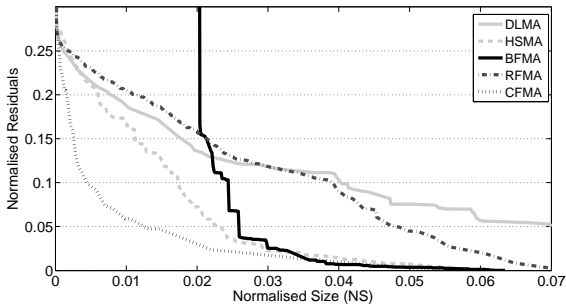

(a) Ray

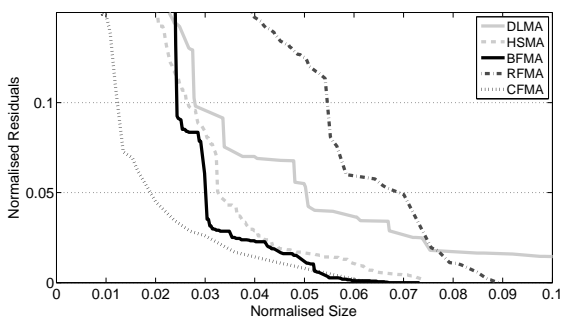

(c) Fountain

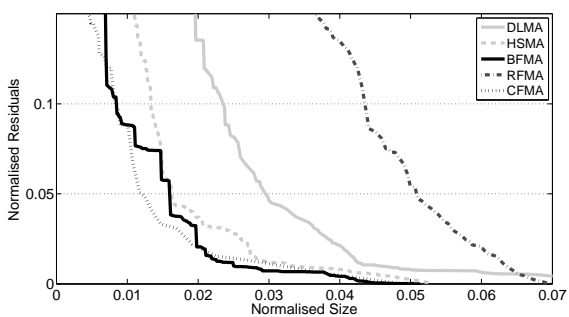

(e) Misk

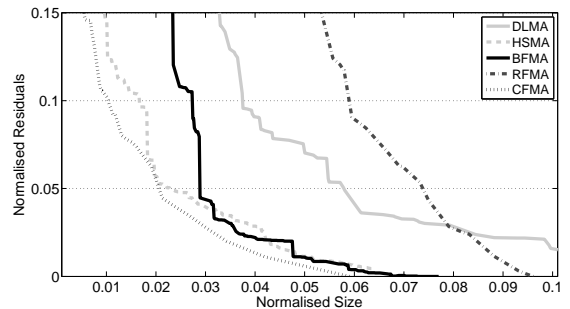

(b) Elephant

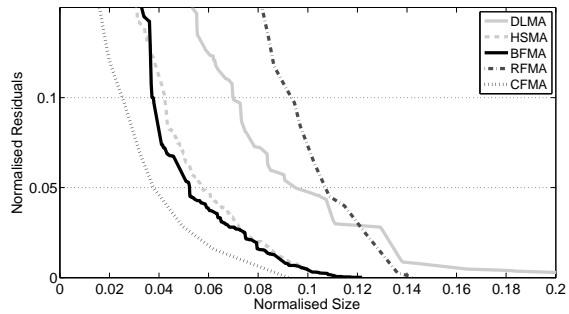

(d) Camel

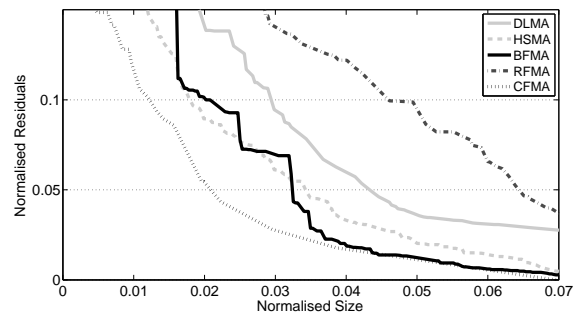

(f) Classic

Figure 12: Residuals as a function of normalised medial axis size for different filtering criteria. Results generated for 6 selected shapes (see Fig 10).

and $d(X \mid Y)=\max \{D(X \mid Y), D(Y \mid X)\}$ is the dissimilarity between $X$ and $Y$.

In Fig. 13 we show the dissimilarity as a function of normalised residuals for different shapes and different filtering methods. The results shows that in all cases BFMA produce lower dissimilarity than other filtering criteria for the same number of normalised residuals. It means that BFMA preserves more visually important features, on the negative side it also preserves some noise. The HSMA produces results comparable to BFMA, but without the same sensitivity to noise. We can also observe a good behaviour of the CFMA up to some level of residuals. After reaching this level it starts removing features 
at low scale which cause dissimilarity to rapidly increase. On the other hand, DLMA and RFMA gives comparable results for the objects without features at different scales, and very poor results for the other ones.

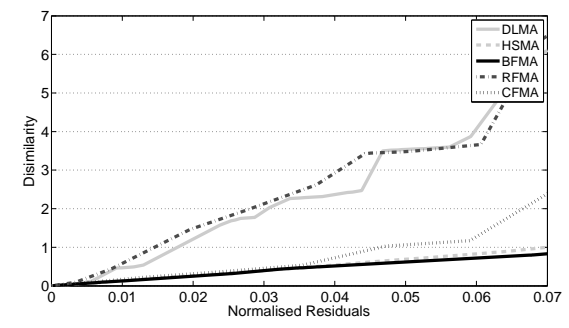

(a) Ray

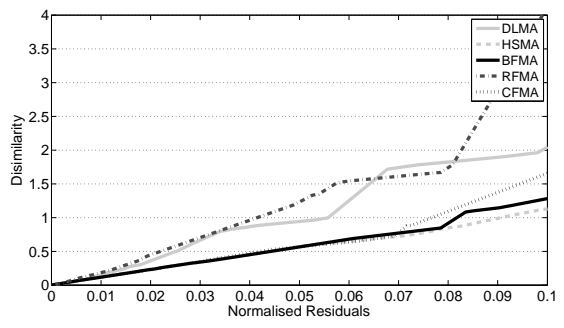

(c) Fountain

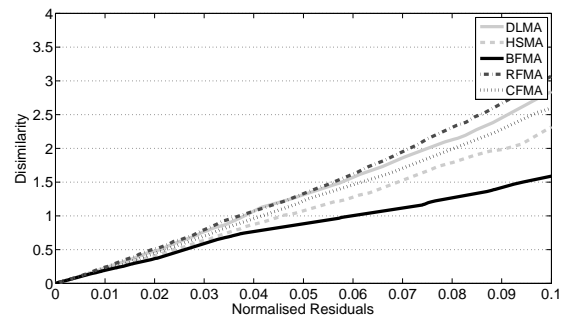

(e) Misk

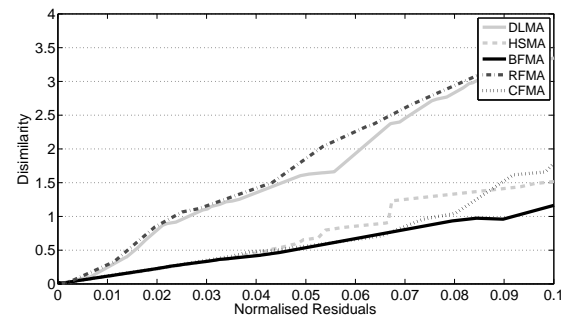

(b) Elephant

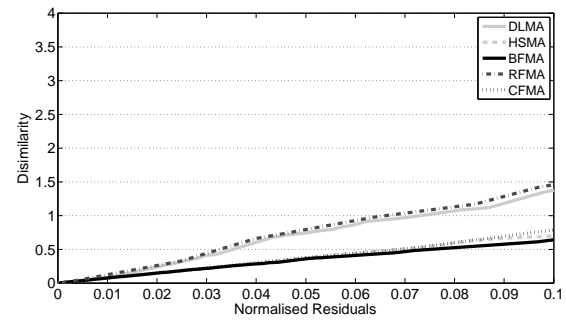

(d) Camel

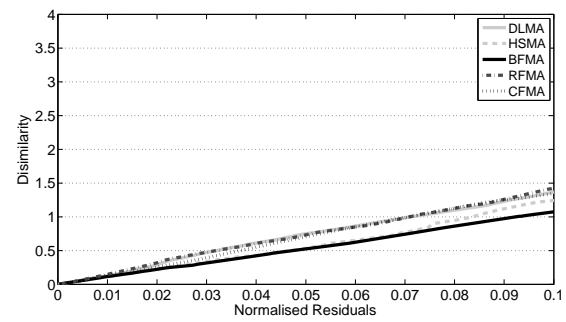

(f) Classic

Figure 13: Dissimilarity as a function of normalised residuals for different filtering criteria. Results generated for 6 selected shapes (see Fig 10).

\subsection{The complexity of the skeleton}

To evaluate the skeleton complexity, we have to consider skeletons that have the same topological characteristics as the original objects. In order to guarantee topology preservation, we perform an ultimate homotopic thinning of $X$ with the constraint of retaining the points of its filtered medial axis $Y$ 
(constraint set), that is, we iteratively remove simple points from $X$ that do not belong to $Y$ (see Fig. 14). A priority function is needed in order to specify which points must be considered at each step of the thinning. In the general case, the choice of this priority function is not obvious as reported in $[16,7]$. For example, taking the exact Euclidean distance map as a priority function may cause "extra branches" to appear which change the skeleton complexity and bias the comparison. Taking this fact into consideration we choose the function $\mathcal{F}_{X}$ defined in [5] as priority.

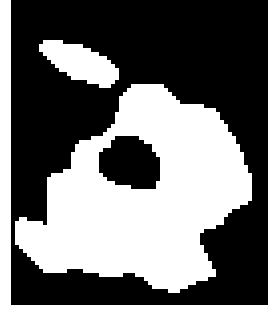

(a)

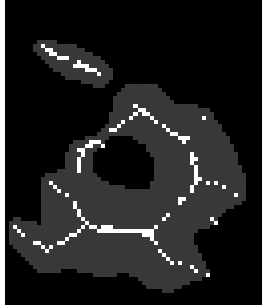

(b)

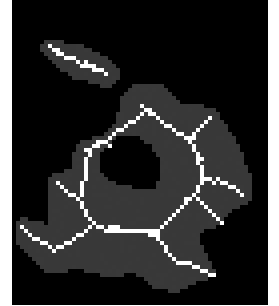

(c)

Figure 14: (a): a set $X$ (in white); (b): medial axis of $X$; (c): ultimate homotopic skeleton of $X$ constrained by the medial axis of $X$. In (b,c) the original set $X$ appears in grey for comparison.

Now, we define the complexity of the skeleton as the number of its end points. Notice that, due to the preservation of the connectivity, any isolated medial axis point that corresponds to unfiltered noise on the border of the object, will generate a spurious branch of the skeleton (see Fig. 14).

We propose another comparison strategy which relies on this notion od skeleton complexity. Quite often in applications, the desired degree of filtering can be expressed in terms of skeleton complexity (for example, the shape of a human silhouette should be represented by a skeleton of complexity five, with end points in the head, the arms and the legs). To each number of end points of the successive filtered skeletons, corresponds a degree of filtering that we evaluate quantitatively by its reconstruction error.

For a given method we denote by $Y(X, p)$ the filtered medial axis at value $p$ of the parameter. To each of the sets $Y(X, p)$ corresponds a homotopic skeleton $S(Y(X, p))$ that has a certain complexity. Note that, for a given complexity $c$, there may exist several values of $p$, or none, such that the complexity of $S(Y(X, p))$ is $c$.

Now for a given complexity $c$, we consider the largest (in the sense of 
the inclusion) set $Y(X, p)$ such that the complexity of $S(Y(X, p))$ is $c$. We denote by $Y_{c}(X)$ this set, or $\emptyset$ if it does not exist. The quality of this filtered medial axis will be evaluated by computing $R_{X}\left(Y_{c}(X)\right)$, that is, the residuals of $Y_{c}(X)$.

Now we can simply compare medial axes at each complexity level independently by changing the parameter $c$ without caring about meaning of the filtering parameter $p$. We could also make a comparison by focusing only on the most suitable level for the shapes of interest. For example if we consider shapes similar to the letters "p", "b", "d", etc., the most interesting complexity level is one, because these shapes have one visually important branch. Therefore, to compare filtered medial axes of such family of shapes we would use the value of $R_{X}\left(Y_{1}(X)\right)$.

Figure 15 shows the minimum residuals at different complexity levels. We can notice that HSMA gives the lowest residual at the most suitable complexity according to the shape. The HSMA gives poor results only for very low complexity, since the method very well preserves features at different scales. Comparable results are generated by CFMA. The BFMA has the worst behaviour in this experiment, since it preserves many points which are close to the border. Therefore, the BFMA is unable to generate medial axes with low complexity. This strongly limits its practical application.

\subsection{The richness of the multiscale representation}

The last interesting criterion is related to the hierarchy property of the medial axes (see definition 2 in section 5). In some applications, one can be more interested in a filtering strategy which can not only remove large amounts of points at once, but also allows one to produce some intermediate results at different levels of hierarchy. Then, the different methods may be compared on the basis of their ability to propose a rich set of hierarchy levels. This is also an important criterion if, in the end, only one filtered axis is kept, for its choice can be done more finely if the number of possibilities is larger.

Let us denote by $M A_{s}(X)$ the filtered medial axis of $X \subset \mathbb{Z}^{n}$ for the value $s$ of the filtering parameter. Since all methods in our comparison hold the hierarchy property, we know that $s>t$ implies $M A_{s}(X) \subseteq M A_{t}(X)$. However, due to the discrete nature of the object $X$, there exists only a finite set of values $s_{0}, \ldots, s_{k}$ yielding distinct filtered axes. More precisely, we consider the set $\left\{s_{i}\right\}_{i=0}^{k}$ of positive real numbers such that:

i) $M A_{s_{0}}(X)=X$, and

ii) $M A_{s_{i}}(X) \neq M A_{s_{i-1}}(X)$, for any $i \in\{1, \ldots, k\}$, and 


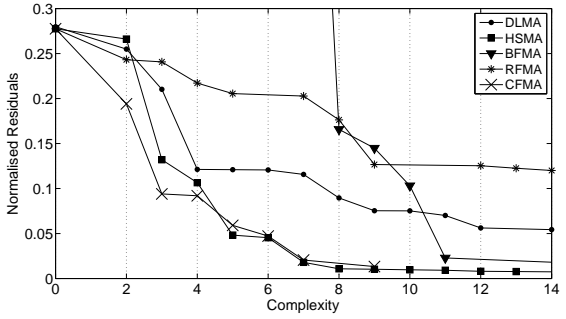

(a) Ray

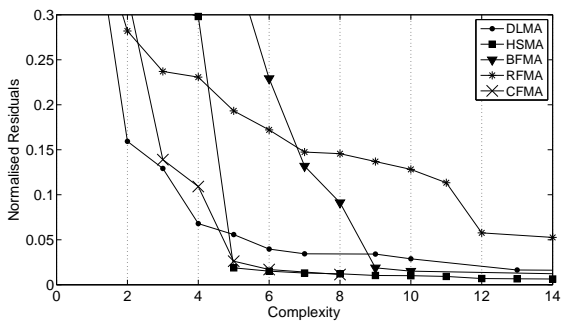

(c) Fountain

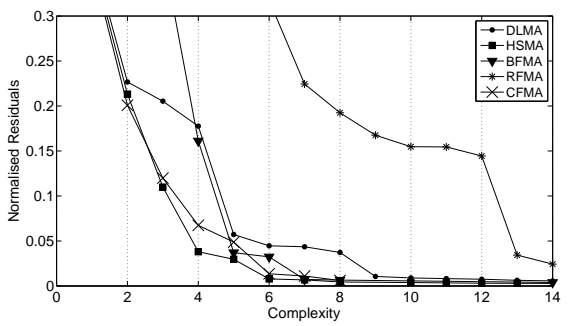

(e) Misk

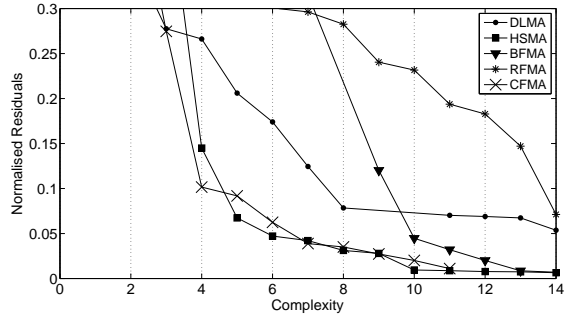

(b) Elephant

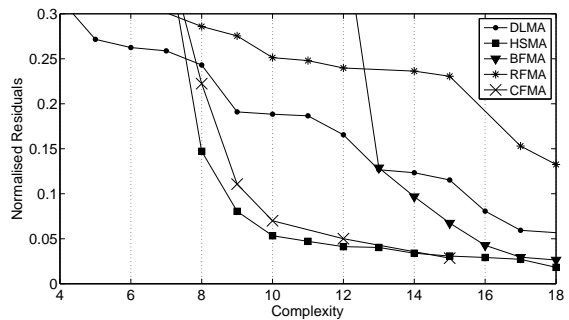

(d) Camel

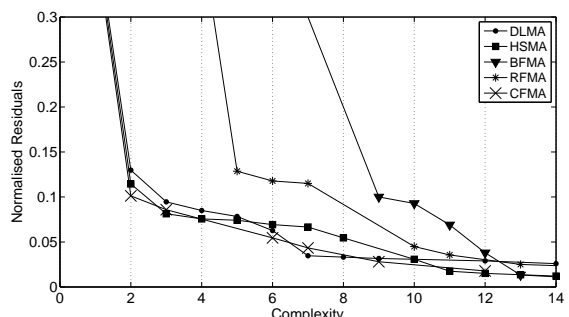

(f) Classic

Figure 15: Normalized residuals in function of the complexity level, calculated for different filtering methods. Results have been generated for the 6 selected shapes (see Fig 10). Each marker (e.g. triangle, circle and square) represents a method. Lines have been added only to emphasize the trend of measurements.

iii) $M A_{s_{i}}(X)=M A_{s}(X)$, for any $i \in\{0, \ldots, k-1\}$ and any $s \in\left[s_{i}, s_{i+1}[\right.$. In the following, the indices $i$ are called the hierarchy levels.

Then, we can evaluate the reconstruction error at each hierarchy level independently. When we increase the hierarchy level, the filtering algorithm removes a set of medial axis points with the same "importance". The "importance" of the point depends on the filtering criterion. The filtering method which removes a smaller amount of points at each change of hierarchy level, can produce more different representations of the object. 
In Fig. 16, we show achieved numbers of normalised residuals at each hierarchy level for all tested filtering methods and for different shapes. Based on these results, we can observe how many different medial axes can be produced by manipulating the filtering parameter and how the reconstruction error evolves.

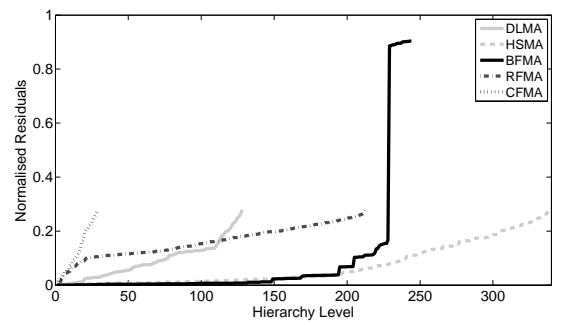

(a) Ray

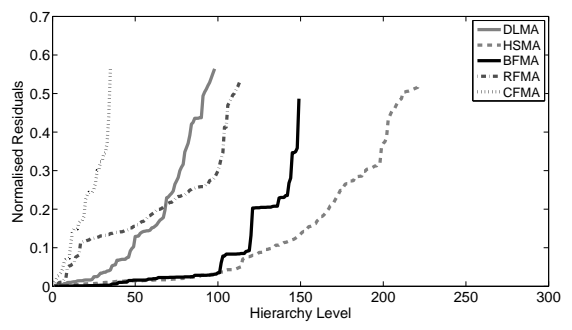

(c) Fountain

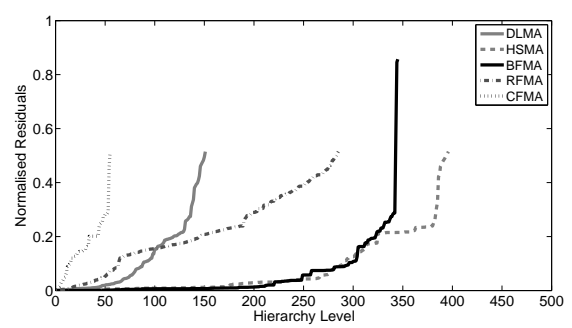

(e) Misk

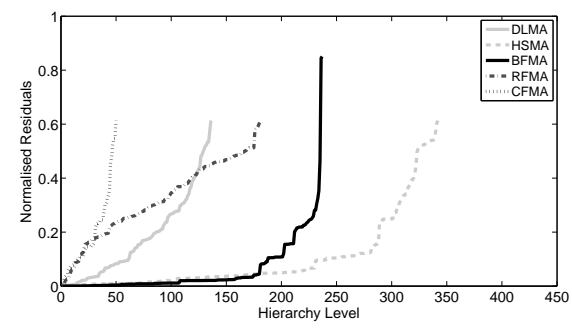

(b) Elephant

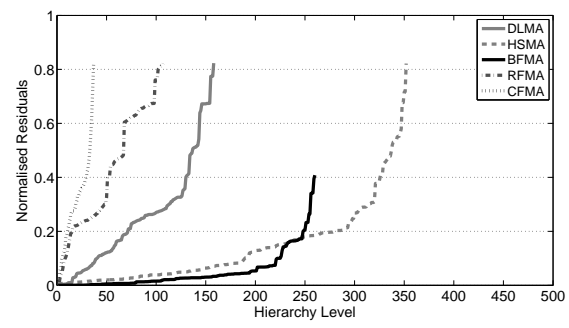

(d) Camel

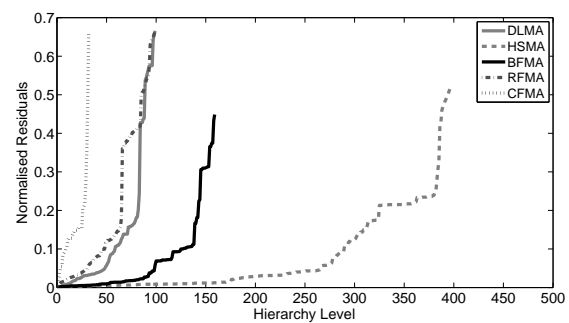

(f) Classic

Figure 16: Residuals as a function of the hierarchy level, for different filtering criteria. Results were generated for the 6 shapes of Fig 10.

For the first four objects which have many features at different scales, the HSMA generates the highest number of multiscale representations of the object. For the last two classes of objects HSMA gives globally the best result. In contrast, the CFMA yields globally the smallest number of 
hierarchy levels. RFMA and DLMA also generate a relatively small number of medial axes. Indeed, the number of different filtered medial axes generated with CFMA, RFMA and DLMA might be not enough in some applications.

\subsection{Discussion}

The usefulness of each filtering method is strongly application dependent. Each of the presented experiments gives us some information about which filtering method is best in respect to the evaluated property. However, each experiment alone is not sufficient to clearly show which one filtering method is the best one at a global scope. In addition, some of evaluated properties are not compatible. For example, at the same time we would like to minimize the reconstruction error, which at the extreme leads to keep most of the medial axis points, but we also to get skeletons with a low complexity, and get rid of points that correspond to noise or useless details. Such incompatibility is very well visible on the results achieved by BFMA, where for medial axes of small size BFMA gives the lowest dissimilarity. Unfortunately, the complexity of the skeletons induced by BFMA is very high.

To objectively compare different filtering criteria at a global scope, we need to look at results of all experiments at the same time. We can see that HSMA yields the best or comparable results to the best one in all experiments. Moreover, HSMA permits to produce a large set of hierarchy levels, which makes it very flexible. We can say that HSMA give us the best balance between the reconstruction error, the size of medial axis the complexity, the richness of the multiscale representation, and allow one to preserve features at different scales. Therefore, HSMA can be considered, globally, as the best method in our comparison.

We can also notice the good behaviour of the CFMA. Only the lowest number of possible hierarchy levels, and high dissimilarity for larger residuals can limit its application. If such features are irrelevant according to application, then CFMA is a good choice.

In our comparison, DLMA and RFMA give the worst results in our experiments. This is not surprising for both methods, since the filtering parameter is defined and acts globally. However, in contrast to RFMA, which is the simplest method and in practice is used only as a supplementary criterion, DLMA holds other interesting properties which are out of scope of this comparison. For example, stability to noise or rotation, makes DLMA very useful in certain practical applications (see $[27,5]$ for a comprehensive evaluation of different DLMA properties). 


\section{Conclusions}

The main outcomes of the article are three contributions. The first contribution is a new method for Euclidean medial axis filtering which possesses the following properties:

- it generates subsets of the Euclidean medial axis,

- the filtering is based on only one parameter,

- it generates filtered medial axes which preserve important parts of an input object at different scales,

- it allows for a better reconstruction of the input object, compared to other related medial axis filtering algorithms,

- the computation complexity of the algorithm is $O(\# X \# M A(X))$,

- it extends trivially to any finite dimension.

The second and the most important contribution, is an algorithm which, for any $X \subseteq Z^{2}$, generates a full hierarchy of scale filtered medial axes (HSMA) in time $O(\# X \# M A(X))$. This algorithm also trivially extends for the case of $Z^{n}$. We prove that the algorithm indeed computes the HSMA as we defined it.

The third contribution consists of a new methodology which allows for a fair comparison of medial axis filtering algorithms. The methodology is based on five different quality criteria. Using this new methodology, one can compare quantitatively properties of algorithms, independently of the meaning of specific algorithm parameters. Following this methodology, we made a series of experiments that show that our algorithm to compute the HSMA gives better or similar results, compared to the best related methods.

\section{Acknowledgements}

This work has been partially supported by the "ANR-2010-BLAN-0205 KIDICO" project. 


\section{References}

[1] H. Blum, A transformation for extracting new descriptors of shape, in: Models for the Perception of Speech and Visual Form, MIT Press, 1967, pp. 362-380.

[2] G. Borgefors, I. Ragnemalm, S. di Baja, The euclidean distance transform: finding the local maxima and reconstructing the shape, in: Procs. of the 7th Scand. Conf. on image analysis, Vol. 2, 1991, pp. 974-981.

[3] W. Hesselink, J. Roerdink, Euclidean skeletons of digital image and volume data in linear time by the integer medial axis transform, IEEE Trans. on PAMI 30 (12) (2008) 2204-2217.

[4] J. Hulin, Axe médian discret : Propriétés arithmétiques et algorithmes, Ph.D. thesis, Université Aix-Marseille II, Marseille (2009).

[5] J. Chaussard, M. Couprie, H. Talbot, Robust skeletonization using the discrete lambda-medial axis, Pattern Recognition Letters 32 (9) (2011) $1384-1394$.

[6] E. R. Davies, A. P. N. Plummer, Thinning algorithms: A critique and a new methodology, Pattern Recognition 14 (1-6) (1981) 53-63.

[7] H. Talbot, L. Vincent, Euclidean skeletons and conditional bisectors, in: Proceedings of VCIP'92, SPIE, Vol. 1818, 1992, pp. 862-876.

[8] J. Serra, Image analysis and mathematical morphology, Academic Press, 1982.

[9] D. Attali, J. Lachaud, Delaunay conforming iso-surface, skeleton extraction and noise removal, Computational Geometry: Theory and Applications 19 (2001) 175-189.

[10] R. Ogniewicz, O. Kübler, Hierarchic voronoi skeletons, Pattern Recognition 28 (33) (1995) 343-359.

[11] K. Siddiqi, S. Bouix, A. Tannenbaum, S. Zucker, The hamilton-jacobi skeleton, in: International Conference on Computer Vision (ICCV), 1999, pp. 828-834. 
[12] R. Kimmel, D. Shaked, N. Kiryati, A. M. Bruckstein, Skeletonization via distance maps and level sets, Computer Vision and Image Understanding 62 (1995) 382-391.

[13] M. Couprie, G. Bertrand, Discrete topological transformations for image processing, in: V. E. Brimkov, R. P. Barneva (Eds.), Digital Geometry Algorithms, Vol. 2 of Lecture Notes in Computational Vision and Biomechanics, Springer, 2012, Ch. 3, pp. 73-107.

[14] D. Attali, J.-D. Boissonnat, H. Edelsbrunner, Stability and computation of the medial axis - a state-of-the-art report, in: Mathematical Foundations of Scientific Visualization, Computer Graphics, and Massive Data Exploration, Springer-Verlag, 2009, pp. 109-125.

[15] D. Attali, G. Sanniti di Baja, E. Thiel, Pruning discrete and semicontinuous skeletons, in: Conference on Image Analysis and Processing, Vol. 974 of LNCS, Springer, 1995, pp. 488-493.

[16] M. Couprie, D. Coeurjolly, R. Zrour, Discrete bisector function and euclidean skeleton in 2d and 3d, Image Vision Comput. 25 (10) (2007) $1543-1556$.

[17] F. Chazal, A. Lieutier, The $\lambda$-medial axis, Graphical Models 67 (4) (2005) 304-331.

[18] D. Coeurjolly, A. Montanvert, Optimal separable algorithms to compute the reverse euclidean distance transformation and discrete medial axis in arbitrary dimension, IEEE Trans. Pattern Anal. Mach. Intell. 29 (3) (2007) 437-448. doi:http://dx.doi.org/10.1109/TPAMI.2007.54.

[19] B. Miklos, J. Giesen, M. Pauly, Discrete scale axis representations for 3d geometry, ACM Trans. Graph. 29 (2010) 101:1-101:10. doi:http://doi.acm.org/10.1145/1778765.1778838.

[20] J. Giesen, B. Miklos, M. Pauly, C. Wormser, The scale axis transform, in: Proceedings of the 25th annual symposium on Computational geometry, SCG '09, ACM, New York, NY, USA, 2009, pp. 106-115. doi:http://doi.acm.org/10.1145/1542362.1542388. 
[21] M. Postolski, M. Couprie, M. Janaszewski, Scale filtered euclidean medial axis, in: R. Gonzalez-Diaz, M.-J. Jimenez, B. Medrano (Eds.), Discrete Geometry for Computer Imagery, Vol. 7749 of Lecture Notes in Computer Science, Springer Berlin Heidelberg, 2013, pp. 360-371.

[22] J. Chassery, A. Montanvert, Géometrie discrète, Hermès, 1991.

[23] T. Y. Kong, A. Rosenfeld, Digital topology: Introduction and survey, Computer Vision, Graphics, and Image Processing 48 (3) (1989) 357393.

[24] A. Rosenfeld, Digital image processing, Academic Press, 1982.

[25] T. H. Cormen, C. Leiserson, R. Rivest, Introduction to algorithms, MIT Press, 1990.

[26] D. Sharvit, J. Chan, H. Tek, B. Kimia, Symmetry-based indexing of image databases, Journal of Visual Communication and Image Representation 9 (4) (1998) 366-380.

[27] J. Chaussard, Topological tools for discrete shape analysis, Ph.D. thesis, Université Paris-Est (December 2010).

[28] M. v. Eede, D. Macrini, A. Telea, C. Sminchisescu, S. S. Dickinson, Canonical skeletons for shape matching, in: Proceedings of the 18th International Conference on Pattern Recognition - Volume 02, ICPR '06, IEEE Computer Society, Washington, DC, USA, 2006, pp. 64-69. doi:http://dx.doi.org/10.1109/ICPR.2006.354.

[29] M. Postolski, Discrete topology and geometry algorithms for quantitative human airway trees analysis based on computed tomography images, Ph.D. thesis, Université Paris-Est and Eódź University of Technology (December 2013). 